\title{
Correction to: Characterizing Pain Points in Clinical Data Management and Assessing the Impact of Mid-Study Updates
}

Beth Harper, MBA ${ }^{1} \cdot$ Zachary Smith, MA ${ }^{2} \oplus$ - Jane Snowdon, PhD, FAMIA ${ }^{3}$. Robert DiCicco, PharmD ${ }^{3}$. Rezzan Hekmat, $\mathrm{MSH}^{3} \cdot$ Van Willis, $\mathrm{PhD}^{3}$. Dilhan Weeraratne, $\mathrm{PhD}^{3} \cdot$ Ken Getz, $\mathrm{MBA}^{2}$

Published online: 17 May 2021

(c) The Drug Information Association, Inc 2021

Correction to: Therapeutic Innovation \& Regulatory Science https://doi.org/10.1007/s43441-021-00301-z

The original article has been corrected due to typographical errors in the Abstract.

The original article can be found online at https://doi.org/10.1007/ s43441-021-00301-z.

Zachary Smith

Zachary.Smith605922@tufts.edu

1 Clinical Performance Partners, Aurora, IL, USA

2 Tufts CSDD, 75 Kneeland Street- Floor 11, Boston, MA,

USA

3 IBM Watson Health, Cambridge, MA, USA 